\title{
Cylindrical thin-shell wormholes
}

\author{
Ernesto F. Eiroa ${ }^{1, *}$, Claudio Simeone $e^{1,2, \dagger}$ \\ ${ }^{1}$ Instituto de Astronomía y Física del Espacio, C.C. 67, Suc. 28, 1428, Buenos Aires, Argentina \\ 2 Departamento de Física, Facultad de Ciencias Exactas y Naturales, \\ Universidad de Buenos Aires, Ciudad Universitaria Pab. I, 1428, Buenos Aires, Argentina
}

November 15, 2018

\begin{abstract}
A general formalism for the dynamics of non rotating cylindrical thin-shell wormholes is developed. The time evolution of the throat is explicitly obtained for thin-shell wormholes whose metric has the form associated to local cosmic strings. It is found that the throat collapses to zero radius, remains static or expands forever, depending only on the sign of its initial velocity.
\end{abstract}

PACS number(s): 04.20.Gz, 11.27.+d, 04.40.Nr

Keywords: Lorentzian wormholes; exotic matter; cosmic strings

\section{Introduction}

Traversable Lorentzian wormholes, first studied by Morris and Thorne [1, are solutions of the Einstein field equations that have two regions connected by a throat. These tunnels can join two parts of the same universe or two separate universes [1, 2. For static wormholes, the throat is defined as a two-dimensional hypersurface of minimal area that should satisfy a flare-out condition [3]. For time dependent wormholes the general definition of the throat is more complex (the interested reader is referred to [4]). All traversable wormholes must be threaded by exotic matter that violates the null energy condition [1, 2, 3, 4, Recently, Visser et al [5] showed that the amount of exotic matter that must be present around the throat can be made infinitesimally small by a suitable choice of the geometry of the wormhole.

In a gauge theory, spontaneous symmetry breaking of a complex scalar field leads to cylindrical topological defects known as local cosmic strings [6]. The gravitational effects of such objects have been the object of thorough analysis, because of the possible important consequences they could have had for galaxy formation, and also in the study of gravitational lensing. The spacetime metric around a local cosmic string was first obtained by Vilenkin [7] in the linear approximation of general relativity. Local strings are characterized by having an energy-momentum tensor whose only non null components are $T_{t}^{t}=T_{z}^{z}$. Within this framework a Dirac's delta was used to model the radial distribution of the energy-momentum tensor for a string along the $z$ axis. The resulting spacetime metric is flat but with a deficit angle $\Delta \varphi=8 \pi G \mu$, up to first order in $G \mu$ (in GUT strings $G \mu \sim 10^{-6}$ ), with $\mu$ the linear energy density. Later, independently, Gott [8] and Hiscock 9], extending the analysis to the framework of theories leading to values of $G \mu$ closer to

*e-mail: eiroa@iafe.uba.ar

†e-mail: csimeone@df.uba.ar 
one, showed that the deficit angle found by Vilenkin is actually right to all orders in $G \mu$. In their demonstration, they considered a source in the form of a thick cylinder of constant radius with both uniform linear mass density and tension, the last one only along the axis. The solution of the full Einstein equations in the interior was matched with the vacuum solution for the exterior, and the integration constants appearing were determined by matching both metrics in the boundary. In all these works, the source determined an exterior solution with Lorentz invariance along the $z$ axis. However, if this is not required, the most general solution has the well-known Weyl form; which includes two solutions with Lorentz invariance, one of them corresponding to the case solved by Gott and Hiscock. Later, similar analysis were extended to scalar tensor theories of gravity, like that of Brans and Dicke [10. It has also been shown [11] that a cosmic string can behave as a superconductor, with a current along it and a magnetic field in its exterior. This can be the result of the appearance of both bosonic or fermionic charge carriers. The spacetime around a superconducting string is then not a vacuum solution of the Einstein equations, because, besides the effective mass associated with the charge carriers, in this case there is a non localized magnetic contribution to the energy-momentum tensor [12.

Solutions of the Einstein field equations representing wormholes associated with cosmic strings have been previously considered in the literature. Clément 13] found traversable multi-wormhole solutions where the spacetime metric was asymptotic to the conical cosmic string metric. In other work, Clément [14] extended cylindrical multi-cosmic strings metrics to wormhole spacetimes with only one region at spatial infinite, and analyzed in detail the geometry of asymptotically flat wormhole spacetimes produced by one or two cosmic strings. Aros and Zamorano [15] constructed a solution that can be interpreted as a traversable cylindrical wormhole inside the core of a global cosmic string.

Thin-shell wormholes are made by cutting and pasting two manifolds [2, 16] to form a geodesically complete new one with a throat placed in the joining shell. In this case, the exotic matter needed to build the wormhole is concentrated at the shell and the junction-condition formalism is used for its study. Poisson and Visser [17] made a linearized stability analysis under spherically symmetric perturbations of a thin-shell wormhole constructed by joining two Schwarzschild geometries. Later, Barceló and Visser [18 applied this method to study wormholes constructed using branes with negative tensions and Ishak and Lake [19] analyzed the stability of transparent spherically symmetric thin-shells and wormholes. Recently, Eiroa and Romero [20] extended the linearized stability analysis to Reisner-Nordström thin-shell geometries, and Lobo and Crawford 21] to wormholes with a cosmological constant.

In this article we study cylindrical thin-shell wormholes. We concentrate on the geometry of these objects and we do not intend to supply any explanation about the mechanisms that might provide the exotic matter to them. In Sec. 2 we present the general formalism. In Sec. 3] and 4, we apply it to vacuum and superconducting cosmic string wormholes. Finally, in Sec. 5] the results are discussed. Throughout the paper we use units such as $c=G=1$.

\section{Cylindrical thin-shell wormholes}

The static cylindrically symmetric metric in coordinates $X^{\alpha}=(t, r, \varphi, z)$ can be written in the form 22 .

$$
d s^{2}=f(r)\left(-d t^{2}+d r^{2}\right)+g(r) d \varphi^{2}+h(r) d z^{2},
$$


where $f, g$ and $h$ are positive functions of $r$. From this geometry we can take two copies ${ }^{1}$ of the region with $r \geq a$ :

$$
\mathcal{M}^{ \pm}=\{x / r \geq a\}
$$

and glue them together at the hypersurface

$$
\Sigma \equiv \Sigma^{ \pm}=\{x / r-a=0\},
$$

to make a geodesically complete manifold $\mathcal{M}=\mathcal{M}^{+} \cup \mathcal{M}^{-}$. If $g_{\varphi \varphi}=g(r)$ is an increasing function for $r \in[a, a+\epsilon]$, with $\epsilon>0$, this construction creates a cylindrically symmetric thin-shell wormhole with two regions connected by a throat at $\Sigma$. On $\mathcal{M}$ we can define a new radial coordinate $l= \pm \int_{a}^{r} g_{r r} d r$, where the positive and negative signs correspond, respectively, to $\mathcal{M}^{+}$and $\mathcal{M}^{-}$, with $|l|$ representing the proper radial distance to the throat, which is placed in $l=0$. To study this traversable wormhole we use the standard Darmois-Israel formalism [23]. For a recent review of this technique, also called junction-condition formalism, see Ref. [24].

The throat of the wormhole is placed at the shell $\Sigma$, which is a synchronous timelike hypersurface. We can adopt coordinates $\xi^{i}=(\tau, \varphi, z)$ in $\Sigma$, with $\tau$ the proper time on the shell. In order to analyze the dynamical behavior, we let the radius of the throat be a function of the proper time, $a=a(\tau)$. Then $\Sigma$ is defined by the equation

$$
\Sigma: \mathcal{H}(r, \tau)=r-a(\tau)=0 .
$$

The extrinsic curvature (second fundamental forms) associated with the two sides of the shell are:

$$
K_{i j}^{ \pm}=-\left.n_{\gamma}^{ \pm}\left(\frac{\partial^{2} X^{\gamma}}{\partial \xi^{i} \partial \xi^{j}}+\Gamma_{\alpha \beta}^{\gamma} \frac{\partial X^{\alpha}}{\partial \xi^{i}} \frac{\partial X^{\beta}}{\partial \xi^{j}}\right)\right|_{\Sigma},
$$

where $n_{\gamma}^{ \pm}$are the unit normals $\left(n^{\gamma} n_{\gamma}=1\right)$ to $\Sigma$ in $\mathcal{M}$ :

$$
n_{\gamma}^{ \pm}= \pm\left|g^{\alpha \beta} \frac{\partial \mathcal{H}}{\partial X^{\alpha}} \frac{\partial \mathcal{H}}{\partial X^{\beta}}\right|^{-1 / 2} \frac{\partial \mathcal{H}}{\partial X^{\gamma}} .
$$

In the orthonormal basis $\left\{e_{\hat{\tau}}, e_{\hat{\varphi}}, e_{\hat{z}}\right\}\left(e_{\hat{\tau}}=\sqrt{1 / f(r)} e_{\tau}, e_{\hat{\varphi}}=\sqrt{1 / g(r)} e_{\varphi}, e_{\hat{z}}=\sqrt{1 / h(r)} e_{z}, g_{\hat{\imath} \hat{\jmath}}=\right.$ $\left.\eta_{\hat{\imath} \hat{\jmath}}=\operatorname{diag}(-1,1,1,1)\right)$ we have

$$
\begin{gathered}
K_{\hat{\tilde{\tau} \hat{\tau}}}^{ \pm}=\mp \frac{2 f(a)^{2} \ddot{a}+f^{\prime}(a)+2 f^{\prime}(a) f(a) \dot{a}^{2}}{2 f(a) \sqrt{f(a)} \sqrt{1+f(a) \dot{a}^{2}}}, \\
K_{\hat{\varphi} \hat{\varphi}}^{ \pm}= \pm \frac{g^{\prime}(a) \sqrt{1+f(a) \dot{a}^{2}}}{2 g(a) \sqrt{f(a)}}
\end{gathered}
$$

and

$$
K_{\hat{z} \hat{z}}^{ \pm}= \pm \frac{h^{\prime}(a) \sqrt{1+f(a) \dot{a}^{2}}}{2 h(a) \sqrt{f(a)}}
$$

where the dot means $d / d \tau$.

The Einstein equations on the shell reduce to the Lanczos equations:

$$
-\left[K_{\hat{\imath} \hat{\jmath}}\right]+[K] g_{\hat{\imath} \hat{\jmath}}=8 \pi S_{\hat{\imath} \hat{\jmath}}
$$

\footnotetext{
${ }^{1}$ It is not necessary to take both regions equal, but it is enough for our purposes.
} 
where $\left[K_{\hat{\imath} \hat{\jmath}}\right] \equiv K_{\hat{\imath} \hat{\jmath}}^{+}-K_{\hat{\imath} \hat{\jmath}}^{-},[K]=g^{\hat{\imath} \hat{\jmath}}\left[K_{\hat{\imath} \hat{\jmath}}\right]$ is the trace of $\left[K_{\hat{\imath} \hat{\jmath}}\right]$ and $S_{\hat{\imath} \hat{\jmath}}=\operatorname{diag}\left(\sigma,-\vartheta_{\varphi},-\vartheta_{z}\right)$ is the surface stress-energy tensor, with $\sigma$ the surface energy density and $\vartheta_{\varphi, z}$ the surface tensions. Then replacing Eqs. (7), (8) and (9) in Eq. (10) we obtain

$$
\begin{gathered}
\sigma=-\frac{\sqrt{1+f(a) \dot{a}^{2}}}{8 \pi \sqrt{f(a)}}\left[\frac{g^{\prime}(a)}{g(a)}+\frac{h^{\prime}(a)}{h(a)}\right], \\
\vartheta_{\varphi}=-\frac{1}{8 \pi \sqrt{f(a)} \sqrt{1+f(a) \dot{a}^{2}}}\left\{2 f(a) \ddot{a}+f(a)\left[\frac{h^{\prime}(a)}{h(a)}+\frac{2 f^{\prime}(a)}{f(a)}\right] \dot{a}^{2}+\frac{h^{\prime}(a)}{h(a)}+\frac{f^{\prime}(a)}{f(a)}\right\}, \\
\vartheta_{z}=-\frac{1}{8 \pi \sqrt{f(a)} \sqrt{1+f(a) \dot{a}^{2}}}\left\{2 f(a) \ddot{a}+f(a)\left[\frac{g^{\prime}(a)}{g(a)}+\frac{2 f^{\prime}(a)}{f(a)}\right] \dot{a}^{2}+\frac{g^{\prime}(a)}{g(a)}+\frac{f^{\prime}(a)}{f(a)}\right\} .
\end{gathered}
$$

The surface energy density is negative, indicating the presence of exotic matter at the throat. The negative signs of the tensions mean that they are indeed pressures.

It is easy to see that $\vartheta_{\varphi}, \vartheta_{z}$ and $\sigma$ satisfy the equation

$$
\vartheta_{\varphi}-\vartheta_{z}=\frac{g(a) h^{\prime}(a)-g^{\prime}(a) h(a)}{g(a) h^{\prime}(a)+g^{\prime}(a) h(a)} \sigma .
$$

The static equations are obtained with $\dot{a}=0$ and $\ddot{a}=0$ in Eqs. (11), (12) and (13):

$$
\begin{gathered}
\sigma=-\frac{1}{8 \pi \sqrt{f(a)}}\left[\frac{g^{\prime}(a)}{g(a)}+\frac{h^{\prime}(a)}{h(a)}\right], \\
\vartheta_{\varphi}=-\frac{1}{8 \pi \sqrt{f(a)}}\left[\frac{h^{\prime}(a)}{h(a)}+\frac{f^{\prime}(a)}{f(a)}\right], \\
\vartheta_{z}=-\frac{1}{8 \pi \sqrt{f(a)}}\left[\frac{g^{\prime}(a)}{g(a)}+\frac{f^{\prime}(a)}{f(a)}\right] .
\end{gathered}
$$

Eqs. (16) and (17) can be recast in the form

$$
\begin{aligned}
& \vartheta_{\varphi}=\alpha(a) \sigma, \\
& \vartheta_{z}=\beta(a) \sigma,
\end{aligned}
$$

with

$$
\begin{aligned}
& \alpha(a)=\frac{g(a)\left[f(a) h^{\prime}(a)+f^{\prime}(a) h(a)\right]}{f(a)\left[g(a) h^{\prime}(a)+g^{\prime}(a) h(a)\right]}, \\
& \beta(a)=\frac{h(a)\left[f(a) g^{\prime}(a)+f^{\prime}(a) g(a)\right]}{f(a)\left[g(a) h^{\prime}(a)+g^{\prime}(a) h(a)\right]} .
\end{aligned}
$$

The functions $f, g$ and $h$ determine the equations of state $\vartheta_{\varphi}(\sigma)$ and $\vartheta_{z}(\sigma)$ of the exotic matter on the shell.

Let us assume that the equations of state for the dynamic case have the same form as in the static one, i.e. that they do not depend on the derivatives of $a(\tau)$, so $\vartheta_{\varphi}(\sigma)$ and $\vartheta_{z}(\sigma)$ are given by Eqs. (18) and (19), with $\alpha$ and $\beta$ of Eqs. (20) and (21). Then, replacing Eqs. (11) and (12) in 
Eq. (18) (or Eqs. (11) and (13) in Eq. (19)), a simple second order differential equation for $a(\tau)$ is obtained

$$
2 f(a) \ddot{a}+f^{\prime}(a) \dot{a}^{2}=0 .
$$

It is easy to see that

$$
\dot{a}(\tau)=\dot{a}\left(\tau_{0}\right) \sqrt{\frac{f\left(a\left(\tau_{0}\right)\right)}{f(a(\tau))}},
$$

satisfies Eq. (22), with $\tau_{0}$ an arbitrary (but fixed) time. Eq. (23) can be put in the form

$$
\sqrt{f(a)} d a=\dot{a}\left(\tau_{0}\right) \sqrt{f\left(a\left(\tau_{0}\right)\right)} d \tau,
$$

that integrating both sides, it gives

$$
\int_{a\left(\tau_{0}\right)}^{a(\tau)} \sqrt{f(a)} d a=\dot{a}\left(\tau_{0}\right) \sqrt{f\left(a\left(\tau_{0}\right)\right)}\left(\tau-\tau_{0}\right) .
$$

The time evolution of the radius of the throat $a(\tau)$ is formally obtained by calculating the integral and inverting Eq. (25).

\section{$3 \quad$ Vacuum cosmic string wormholes}

The most general metric which can be associated to a local vacuum cosmic string has the Weyl's form

$$
d s^{2}=\left(\frac{r}{r_{0}}\right)^{2 d(d-1)}\left(-d t^{2}+d r^{2}\right)+r^{2} W_{0}^{2}\left(\frac{r}{r_{0}}\right)^{-2 d} d \varphi^{2}+\left(\frac{r}{r_{0}}\right)^{2 d} d z^{2},
$$

where $r_{0}$ (a scaling length for the radial coordinate), $W_{0}>0$ and $d$ are constants. We take $d<1$, so $g_{\varphi \varphi}$ is an increasing function of $r$. Then the surface energy density and tensions at the throat are

$$
\begin{gathered}
\sigma=-\frac{\left(\frac{a}{r_{0}}\right)^{-d(d-1)} \sqrt{1+\left(\frac{a}{r_{0}}\right)^{2 d(d-1)} \dot{a}^{2}}}{4 \pi a}, \\
\vartheta_{\varphi}=-\frac{d^{2}\left(\frac{a}{r_{0}}\right)^{-d(d-1)}+\left(\frac{a}{r_{0}}\right)^{d(d-1)}\left[a \ddot{a}+d(2 d-1) \dot{a}^{2}\right]}{4 \pi a \sqrt{1+\left(\frac{a}{r_{0}}\right)^{2 d(d-1)} \dot{a}^{2}}}, \\
\vartheta_{z}=-\frac{(d-1)^{2}\left(\frac{a}{r_{0}}\right)^{-d(d-1)}+\left(\frac{a}{r_{0}}\right)^{d(d-1)}\left[a \ddot{a}+(d-1)(2 d-1) \dot{a}^{2}\right]}{4 \pi a \sqrt{1+\left(\frac{a}{r_{0}}\right)^{2 d(d-1)} \dot{a}^{2}}} .
\end{gathered}
$$

For the static case we have that $\vartheta_{\varphi}=d^{2} \sigma$ and $\vartheta_{z}=(d-1)^{2} \sigma$. Keeping these equations of state for the dynamic case and using Eq. (25), we obtain

$$
\frac{a(\tau)}{r_{0}}=\left\{\left[\frac{a\left(\tau_{0}\right)}{r_{0}}\right]^{d(d-1)+1}+\dot{a}\left(\tau_{0}\right)\left[\frac{a\left(\tau_{0}\right)}{r_{0}}\right]^{d(d-1)}[d(d-1)+1] \frac{\tau-\tau_{0}}{r_{0}}\right\}^{\frac{1}{d(d-1)+1}} .
$$

As $d(d-1)+1$ is positive for all $d$, from Eq. (30) we see that if the initial velocity of the throat $\dot{a}\left(\tau_{0}\right)$ is positive, the radius of the throat increases (without bounds) with time, while in the case 
of negative initial velocity it decreases to collapse to $a=0$ in a finite time, and if $\dot{a}\left(\tau_{0}\right)=0$ the throat has constant radius $a\left(\tau_{0}\right)$ (static solution).

When $d \neq 0$ the geometry outside the throat could be interpreted as the one corresponding to a wiggly or noisy cosmic string. A special interesting case is the straight cosmic string wormhole, which is invariant under boosts in $z$ and corresponds to $d=0$, its metric given by

$$
d s^{2}=-d t^{2}+d r^{2}+W_{0}^{2} r^{2} d \varphi^{2}+d z^{2} .
$$

This geometry is conical with a deficit angle $\Delta \varphi=2 \pi\left(1-W_{0}\right)$ if $0<W_{0}<1$ (surplus angle if $\left.W_{0}>1\right)$. The energy density and tensions are

$$
\begin{gathered}
\sigma=-\frac{\sqrt{1+\dot{a}^{2}}}{4 \pi a}, \\
\vartheta_{\varphi}=-\frac{\ddot{a}}{4 \pi \sqrt{1+\dot{a}^{2}}},
\end{gathered}
$$

and

$$
\vartheta_{z}=-\frac{1+\dot{a}^{2}+a \ddot{a}}{4 \pi a \sqrt{1+\dot{a}^{2}}}
$$

The static solution has $\sigma=\vartheta_{z}=-1 / 4 \pi a$ and $\vartheta_{\varphi}=0$; and the energy density per unit length is $\mu=2 \pi W_{0} a \sigma=-W_{0} / 2$. In the dynamic case, using Eq. (30) with $d=0$, we have

$$
a(\tau)=a\left(\tau_{0}\right)+\dot{a}\left(\tau_{0}\right)\left(\tau-\tau_{0}\right),
$$

so in this case there is a simple linear dependence with time for the radius of throat.

\section{Superconducting cosmic string wormholes}

The exterior metric for a superconducting cosmic string has the form [12]

$$
d s^{2}=\left(\frac{r}{r_{0}}\right)^{-2 m} A^{2}(r)\left[\left(\frac{r}{r_{0}}\right)^{2 m^{2}}\left(-d t^{2}+d r^{2}\right)+W_{0}^{2} r^{2} d \varphi^{2}\right]+\left(\frac{r}{r_{0}}\right)^{2 m} \frac{1}{A^{2}(r)} d z^{2},
$$

where

$$
A(r)=\frac{\left(\frac{r}{r_{0}}\right)^{2 m}+k}{1+k},
$$

with $r_{0}$ (a scaling length for the radial coordinate), $k \geq 0, W_{0}>0$ and $m$ constants. If we take $-1<m<1, g_{\varphi \varphi}$ is an increasing function of $r$ for every (non-negative) value of $k$. The electric current related with this metric is

$$
I= \pm \frac{m W_{0}}{1+k} \sqrt{k}
$$

and the associated magnetic field strength is given by

$$
F_{z r}=-F_{r z}=\frac{ \pm 2 m}{r}\left(\frac{r}{r_{0}}\right)^{2 m}\left[\left(\frac{r}{r_{0}}\right)^{2 m}+k\right]^{-2}(1+k) \sqrt{k} .
$$

With our cut and paste construction, we obtain in this case a wormhole that carries a current $I$ along the throat and a magnetic field outside the throat, given by Eqs. (38) and (39) respectively. 
If $k=0$, there is no current and the magnetic field is zero, and the Weyl's metric is recovered (taking $m=-d$ ).

Using Eqs. (11), (12) and (13), the energy density and tensions at the throat are given by

$$
\begin{aligned}
\sigma= & \frac{-\left(\frac{a}{r_{0}}\right)^{m(1-m)} \sqrt{(1+k)^{2}+\left[\left(\frac{a}{r_{0}}\right)^{2 m}+k\right]^{2}\left(\frac{a}{r_{0}}\right)^{2 m(m-1)}} \dot{a}^{2}}{4 \pi a\left[\left(\frac{a}{r_{0}}\right)^{2 m}+k\right]}, \\
\vartheta_{\varphi}= & \frac{-\left(\frac{a}{r_{0}}\right)^{m(1-m)}}{4 \pi a\left[\left(\frac{a}{r_{0}}\right)^{2 m}+k\right] \sqrt{(1+k)^{2}+\left[\left(\frac{a}{r_{0}}\right)^{2 m}+k\right]^{2}\left(\frac{a}{r_{0}}\right)^{2 m(m-1)} \dot{a}^{2}}} \times \\
& \left\{m^{2}(1+k)^{2}+\left[\left(\frac{a}{r_{0}}\right)^{2 m}+k\right]^{2}\left(\frac{a}{r_{0}}\right)^{2 m(m-1)} a \ddot{a}+\right. \\
& \left.m\left(\frac{a}{r_{0}}\right)^{2 m^{2}}\left[k^{2}(2 m-1)\left(\frac{a}{r_{0}}\right)^{-2 m}+(2 m+1)\left(\frac{a}{r_{0}}\right)^{2 m}+4 m k\right] \dot{a}^{2}\right\},
\end{aligned}
$$

and

$$
\vartheta_{z}=\vartheta_{\varphi}+\left[1+2 m-\frac{4 m k}{\left(\frac{a}{r_{0}}\right)^{2 m}+k}\right] \sigma .
$$

From the static solution we obtain the equations of state

$$
\vartheta_{\varphi}=m^{2} \sigma
$$

and

$$
\vartheta_{z}=\left[(1+m)^{2}-\frac{4 m k}{\left(\frac{a}{r_{0}}\right)^{2 m}+k}\right] \sigma .
$$

Following Sec. 2 we have that the time evolution of the radius of the throat is implicitly given by the equation

$$
\begin{aligned}
\frac{k}{p}\left\{\left[\frac{a(\tau)}{r_{0}}\right]^{p}-\left[\frac{a\left(\tau_{0}\right)}{r_{0}}\right]^{p}\right\}+\frac{1}{q}\left\{\left[\frac{a(\tau)}{r_{0}}\right]^{q}-\left[\frac{a\left(\tau_{0}\right)}{r_{0}}\right]^{q}\right\}= & \dot{a}\left(\tau_{0}\right)\left[\frac{a\left(\tau_{0}\right)}{r_{0}}\right]^{m(m-1)} \times \\
& \left\{k+\left[\frac{a\left(\tau_{0}\right)}{r_{0}}\right]^{2 m}\right\} \frac{\tau-\tau_{0}}{r_{0}},
\end{aligned}
$$

with $p \equiv m^{2}-m+1$ and $q \equiv m^{2}+m+1$ positive numbers for all values of $m$, and $\tau_{0}$ an arbitrary (fixed) time. In all cases the Eq. (45) can be inverted numerically, and in some cases analytically, to obtain $a(\tau)$. 
The velocity and the acceleration of the throat are, respectively,

$$
\dot{a}(\tau)=\dot{a}\left(\tau_{0}\right)\left[\frac{a\left(\tau_{0}\right)}{a(\tau)}\right]^{m(m-1)} \frac{\left[\frac{a\left(\tau_{0}\right)}{r_{0}}\right]^{2 m}+k}{\left[\frac{a(\tau)}{r_{0}}\right]^{2 m}+k},
$$

and

$$
\ddot{a}(\tau)=\frac{-m \dot{a}\left(\tau_{0}\right)^{2}}{a(\tau)}\left\{\frac{\left[\frac{a\left(\tau_{0}\right)}{r_{0}}\right]^{2 m}+k}{\left[\frac{a(\tau)}{r_{0}}\right]^{2 m}+k}\right\}^{2}\left[\frac{a\left(\tau_{0}\right)}{a(\tau)}\right]^{2 m(m-1)}\left\{k(m-1)+(m+1)\left[\frac{a(\tau)}{r_{0}}\right]^{2 m}\right\} .
$$

It is easy to see that the sign of the velocity is given by the sign of the initial velocity $\dot{a}\left(\tau_{0}\right)$ and the acceleration is always negative. As a consequence, if the initial velocity is positive, the throat expands forever, with decreasing velocity, whether in the case of negative initial velocity it contracts to zero radius with increasing (in modulus) velocity. In the case of null initial velocity the radius of the throat remains constant.

There are two limiting cases of interest, which correspond to small values of the current $I$, where $a(\tau)$ can be approximately given in an explicit form. For $k \ll 1$ Eq. (45) gives

$$
\frac{a(\tau)}{r_{0}} \approx\left\{\left(\frac{a\left(\tau_{0}\right)}{r_{0}}\right)^{q}+\dot{a}\left(\tau_{0}\right)\left(\frac{a\left(\tau_{0}\right)}{r_{0}}\right)^{m(m-1)}\left[k+\left(\frac{a\left(\tau_{0}\right)}{r_{0}}\right)^{2 m}\right] q \frac{\tau-\tau_{0}}{r_{0}}\right\}^{1 / q},
$$

and if $k \gg 1$ we obtain

$$
\frac{a(\tau)}{r_{0}} \approx\left\{\left(\frac{a\left(\tau_{0}\right)}{r_{0}}\right)^{p}+\dot{a}\left(\tau_{0}\right)\left(\frac{a\left(\tau_{0}\right)}{r_{0}}\right)^{m(m-1)}\left[1+\frac{1}{k}\left(\frac{a\left(\tau_{0}\right)}{r_{0}}\right)^{2 m}\right] p \frac{\tau-\tau_{0}}{r_{0}}\right\}^{1 / p} .
$$

In both Eqs. (48) and (49), we observe that, as in the case of vacuum cosmic string wormholes, the radius of the throat behaves like a positive power of $\tau$ for non vanishing initial velocity.

\section{Discussion}

In this paper we have developed a general analysis of the dynamics of cylindrical thin-shell wormholes, under a reasonable assumption regarding the equations of state that relate the tensions with the surface energy density of the exotic matter at the throat. The temporal evolution of the radius of the throat was obtained for the general case. We applied this formalism to cylindrical geometries of interest that appear in the context of local cosmic strings. An observer outside the throat would not distinguish the geometry from that of the exterior of a local cosmic string. For the examples studied, corresponding to vacuum and superconducting cosmic string wormholes, we found that the temporal evolution of the throat depends mainly on its initial velocity: if it is positive the throat expands indefinitely, in the negative case it collapses to null radius in a finite time, and when it is zero, the radius of the throat remains constant. In these examples, oscillatory solutions are not possible. There exists a static solution for each value of the throat radius, but these solutions are unstable under perturbations in the velocity, i.e. instead of oscillating around or damping towards an equilibrium position, they collapse or expand forever if a non zero initial velocity is given. Indeed, from Eq. (23), the sign of the initial velocity completely determines the sign of the velocity 
at any time, this being a general feature of cylindrical thin-shell wormholes under the hypothesis of this work. It is easy to see from Eq. (22) that in the general case the acceleration has the opposite sign of the derivative of $g_{r r}=f(r)$, so depending on the metric considered, it would accelerate or decelerate the expansion or contraction of the throat, but without changing the sign of the velocity, which is given by its initial value.

\section{Acknowledgments}

This work has been supported by Universidad de Buenos Aires (UBACYT X-143, E.F.E.) and Universidad de Buenos Aires and CONICET (C.S.). Some calculations in this paper were done with the help of the package GRTensorII [25].

\section{References}

[1] M.S. Morris and K.S. Thorne, Am. J. Phys. 56, 395 (1988).

[2] M. Visser, Lorentzian Wormholes (AIP Press, New York, 1996).

[3] D. Hochberg and M. Visser, Phys. Rev. D 56, 4745 (1997).

[4] D. Hochberg and M. Visser, Phys. Rev. Lett. 81, 746 (1998); D. Hochberg and M. Visser, Phys. Rev D 58, 044021 (1998).

[5] M. Visser, S. Kar and N. Dadhich, Phys. Rev. Lett. 90, 201102 (2003).

[6] A. Vilenkin, Phys. Rep. 121, 263 (1985); A. Vilenkin and E.P.S. Shellard, Cosmic Strings and Other Topological Defects (Cambridge University Press, Cambridge, 1994).

[7] A. Vilenkin, Phys. Rev. D 23, 852 (1981).

[8] J.R. Gott III, Astrophys. J. 288, 422 (1985).

[9] W.A. Hiscock, Phys. Rev. D 31, 3288 (1985).

[10] A.A. Sen, N. Banerjee and A. Banerjee, Phys. Rev. D 56, 3706 (1997); A. Barros and C. Romero, J. Math. Phys. 36, 5800 (1995); A. Arazi and C. Simeone, Gen. Relativ. Gravit. 32, 2259 (2000); A. Arazi and C. Simeone, Mod. Phys. Lett. A 15, 1369 (2000).

[11] E. Witten, Nucl. Phys. B249, 557 (1985).

[12] A. Babul, T. Piran and D.N. Spergel, Phys. Lett. B 202, 307 (1988); A. Babul, T. Piran and D.N. Spergel, Phys. Lett. B 209, 477 (1988).

[13] G. Clément, Phys. Rev. D 51, 6803 (1995).

[14] G. Clément, J. Math. Phys. 38, 5807 (1997).

[15] R.M. Aros and N. Zamorano, Phys. Rev. D 56, 6607 (1997).

[16] M. Visser, Phys. Rev. D 39, 3182 (1989); M. Visser, Nucl. Phys. B328, 203 (1989).

[17] E. Poisson and M. Visser, Phys. Rev. D 52, 7318 (1995).

[18] C. Barceló and M. Visser, Nucl. Phys. B584, 415 (2000). 
[19] M. Ishak and K. Lake, Phys. Rev. D 65, 044011 (2002).

[20] E.F. Eiroa and G.E. Romero, Gen. Relativ. Gravit. 36, 651 (2004).

[21] F.S.N. Lobo and P. Crawford, Class. Quantum Grav. 21, 391 (2004).

[22] K.S. Thorne, Phys. Rev. 138, B251 (1965).

[23] N. Sen, Ann. Phys. (Leipzig) 73, 365 (1924); K. Lanczos, ibid. 74, 518 (1924); G. Darmois, Mémorial des Sciences Mathématiques, Fascicule XXV ch V (Gauthier-Villars, Paris, 1927) ; W. Israel, Nuovo Cimento 44B, 1 (1966); 48B, 463(E) (1967).

[24] P. Musgrave and K. Lake, Class. Quantum Grav. 13, 1885 (1996).

[25] It can be obtained freely at the address http://grtensor.org. 